\title{
CARBAPENEM-RESISTANT ENTEROBACTERIACEAE: NEW MENACE TO OUR ANTIBIOTIC ARMAMENTARIUM!
}

\author{
ANKITA PORWAL, SEVITHA BHAT* \\ Department of Microbiology, Kasturba Medical College, Manipal University, Mangalore, Karnataka, India. Email: sevitha@rediffmail.com
} Received: 28 April 2017, Revised and Accepted: 5 June 2017

ABSTRACT

Objective: To study the risk factors, clinical characteristics, and outcome in patients with infections due to CRE infections.

Methods: Retrospective study was carried out in a microbiology laboratory for 6 months. 152 CRE isolates identified by the Vitek 2 compact system were included in the study. The risk factors, antibiotic treatment and clinical outcome were studied from the case sheets, Chi-square test was used for statistical analysis.

Results: Of the 2520 positive cultures for Enterobacteriaceae, isolation rate of CRE was 152 (6\%). 76 (50\%) belonged to exudates, 22 (14.1\%) blood culture, 90 (59.2\%) urine. The common infection was UTI. The risk factors were prior exposure to antimicrobials, admission to intensive care unit, indwelling devices and prior hospitalization. The effective antibiotic was colistin, polymyxin B, tigecycline, cefoperazone-sulbactum, piperacillintazobactum. The mortality was $19.7 \%$.

Conclusion: We have demonstrated the value of understanding local epidemiology to help modify our risk-based screening as a strategy to limit the spread of CRE.

Keywords: Enterobacteriaceae, Carbapenem-resistance, Risk factors.

(C) 2017 The Authors. Published by Innovare Academic Sciences Pvt Ltd. This is an open access article under the CC BY license (http://creativecommons. org/licenses/by/4. 0/) DOI: http://dx.doi.org/10.22159/ajpcr.2017.v10i9.19448

\section{INTRODUCTION}

The infections caused by Enterobacteriaceae family include urinary tract infections (UTIs), bloodstream infections, hospital-acquired pneumonias and various intra-abdominal infections.

The Enterobacteriaceae are among the most common etiologic agents that cause healthcare-associated infections.

The rise in the number of extended-spectrum beta-lactamases producing Enterobacteriaceae made carbapenems the drug of choice in the treatment of multidrug-resistant (MDR) Enterobacteriaceae. Because of their safety and established efficacy, the carbapenems used to serve as the ultimate last resort option for treating MDR [1].

The family of bacteria known as the carbapenem-resistant Enterobacteriaceae is an emerging group of Gram-negative bacilli that have become resistant to all or nearly all antibiotics and are causing infections with significant morbidity and mortality. The rates of carbapenem resistance have increased over the past decade. The prevalence of carbapenem-resistant Enterobacteriaceae (CRE), according to some institutions in epidemic area, varies between $24.7 \%$ and $29.8 \%[2,3]$.

The risk factors associated with CRE include medical devices such as intravenous catheters, ventilators, urinary catheters, and through wounds caused by injury or surgery. Because these bacteria have become resistant to antibiotics, CRE infections are very difficult to treat.

The emergence of CRE is a menace to patients, particularly to those who are debilitated, with various underlying diseases, complex infections or medical interventions. Moreover, pathogens resistance to carbapenems often shows high resistance to other antibiotic agents as well, such as cephalosporins, quinolones and aminoglycosides, leaving few or, in some cases, no optimal therapeutic options. What's more, some extra factors, such as delayed identification, lack of accurate judgment of pathogens, also lead to high mortality, prolonged hospital stay, and huge medical expenses in CRE-infected patients [4,5].

The study undertaken aims to look into the common Gram-negative bacterial infections mainly of the Enterobacteriaceae family which are resistant to the carbapenems and the etiological agents of these infections. The antibiotic audit in these cases and the risk factor analysis will help us to look into the areas of interventions which can be taken up to prevent the emergence and spread of the CRE.

In this study, we aim to study the risk factors, clinical characteristics, and outcome in patients with infections due to CRE infections.

\section{Objectives}

1. To identify the common infections caused by CRE.

2. To identify the risk factors associated with CRE infections.

3. To study the antibiotic treatment given and clinical outcome in these patients.

\section{MATERIALS AND METHODS}

Study design

Retrospective study.

Study setting

Microbiology laboratory of a tertiary care center. 
Study duration

6 months (June 2016 to November 2016).

\section{Sample size}

The total number of samples received during the 6 months period.

\section{Inclusion criteria}

- Enterobacteriacae isolates from clinically significant samples over a period of 6 months will be included in the study. Blood and cerebrospinal fluid sample having any growth are considered clinically significant. Pus samples and lower respiratory tract aspirates associated with numerous polymorphonuclear cells are considered clinically significant, and urine sample showing growth of more than $10^{5}$ organisms or more than $10^{3}$ organisms in symptomatic patients is considered clinically significant.

- Only the isolates of Enterobacteriaceae which are resistant to carbapenem group of antibiotics will be included in the study.

\section{Exclusion criteria}

- All isolates other than Enterobacteriaceae will be excluded from the study.

- The isolates which are sensitive to carbapenems will be excluded from the study.

\section{Methods}

The CRE isolates from various samples such as pus, urine, blood identified by conventional methods/automated Vitek 2 compact system and the antibiotic susceptibility of the isolates was done by modified Kirby-Bauer disk diffusion method according to Clinical and Laboratory Standards Institute (CLSI) guidelines on Muller-Hinton agar plates. The results were interpreted using CLSI guidelines. The antibiogram was noted.

\section{Data collection}

The clinical details were collected using a pro forma from the case sheets of the patients. Detailed clinical history is to be obtained which will include information such as age, sex, duration of hospital stay, history of chronic disease, history of instrumentation, or device implantation.

The demographics, risk factors, clinical characteristics, antibiotic exposure, and outcome shall be noted. The empirical antibiotic given, any changes/de-escalation done after receiving the report, duration of antibiotic treatment will be noted from the case sheets. The information will be obtained from the medical records department of the hospitals.

\section{Ethical committee clearance}

The present study has been approved by the Institutional Ethics Committee.

\section{Data analysis}

Chi-square test and odds ratio were used to find out association between the different risk factors for CRE was also estimated. The statistical package SPSS version 17.0 was used to perform the analyses.

\section{RESULTS}

Of the 2520 positive cultures for Enterobacteriaceae, isolation rate of CRE was 152 (6\%). Among 152 CRE isolates, 76 (50\%) belonged to exudates, $22(14.1 \%)$ blood culture, and $90(59.2 \%)$ urine. The most common infection was UTI. The findings of the study are shown in Tables 1-6.

\section{Treatment and outcome}

The empirical treatment was started with $3^{\text {rd }}$ generation cephalosporins including ceftriaxone and cefotaxime, carabapenems (meropenem/ imipenem), amoxyclav, amikacin, vancomycin, and piperacillintazobactam. The antibiotic was changed after the antibiotic sensitivity report based on the sensitivity pattern of each organism. The effective antibiotics against the CRE isolates were colistin, polymyxin
B, tigecycline, cefoperazone-sulbactum, piperacillin-tazobactum. Norfloxacin/nitrofurantoin was found to be effective in UTIs.

Of the 152 CRE-infected patients, the mortality rate was 30 (19.7\%). The immediate cause of death in these patients was sepsis with septic shock and multiple organ dysfunction syndrome and the underlying causes were malignancy, cellulitis, and chronic liver disease with portal hypertension.

\section{DISCUSSION}

CRE are an emerging threat and are associated with high mortality. CRE, selected by the use of antibiotics have become very important healthcare-associated pathogens. The risk factors are advanced age, comorbidities, and medical interventions.

The previous studies have reported that pneumonia was the most frequently detected infection (62.07\%), followed by UTIs and central venous catheter-associated bloodstream infections. Prior antimicrobial therapy, urinary catheterization, and length of intensive care unit (ICU) stay were found to be risk factors. Gram-negative Enterobacteriaceae were the most frequently isolated pathogens, while Pseudomonas aeruginosa was the single most frequent causative organism in ICU infections [6].

The prevalence of CRE in our study population was 6\% (152 of 2520 patients), which is almost similar to the prevalence of CRE $5.4 \%$ (306 of 5676 patients) in previous studies done by Swaminathan et al in 2013 [7].

There was a varied range of infections caused by CRE in our hospital set up, such as UTI, sepsis, cellulitis, necrotising fasciitis, pneumonia, and spontaneous bacterial peritonitis which was consistent with the findings of previous studies conducted by Nordmann et al. in 2011 [5]. Interestingly, pneumonia and UTIs were the most common infections in previous research reported by Falagas et al. in the year 2013 [8].

Our study identified similar risk factors that other investigators have highlighted in their studies done in 2014 and 2015 prior exposure to antimicrobials such as $3^{\text {rd }}$ generation cephalosporins, carbapenems and beta-lactam/beta-lactamase inhibitor combinations, admission to ICU, presence to indwelling devices for example urinary catheter, central line, endotracheal tube, and prior hospitalization $[9,10]$. This highlights the importance of safe patient care practices, especially in the care of devices as well as the significance of an antimicrobial stewardship program in the strategy for the prevention of CRE infections.

Infections with CRE result in poor outcomes, and available treatments of the past resort such as tigecycline and colistin are of unclear efficacy and safety [11].

Table 1: Age-wise distribution of CRE isolates

\begin{tabular}{ll}
\hline Age group (years) & Total CRE isolates \\
\hline $0-12$ & 15 \\
$13-25$ & 11 \\
$26-38$ & 15 \\
$39-51$ & 20 \\
$52-64$ & 41 \\
$65-77$ & 37 \\
$78-92$ & 13 \\
\hline
\end{tabular}

CRE: Carbapenem-resistant Enterobacteriaceae

Table 2: Gender-wise distribution of the patients with CRE infections

\begin{tabular}{ll}
\hline Males & Females \\
\hline 88 & 64 \\
\hline CRE: Carbapenem-resistant Enterobacteriaceae &
\end{tabular}


Table 3: Common infections caused and the most common pathogen isolated in Enterobacteriaceae isolates

\begin{tabular}{lll}
\hline Infection caused by CRE isolates & Number of cases & Most common organism isolated \\
\hline UTI & 53 & E. coli, Klebsiella spp. \\
Necrotising fasciitis & 20 & E. coli, Klebsiella spp., Citrobacter spp. \\
Sepsis & 20 & E. coli, Klebsiella spp., Enterobacter spp. \\
Cellulitis & 17 & E. coli, Klebsiella spp., Citrobacter spp. \\
Pneumonia & 34 & Klebsiella spp. \\
Spontaneous bacterial peritonitis & 2 & Klebsiella spp. \\
Organ abscesses & 6 & Klebsiella spp. \\
\hline
\end{tabular}

CRE: Carbapenem-resistant Enterobacteriaceae, UTI: Urinary tract infection, E. coli: Escherichia coli

Table 4: Types of clinical specimen with isolation of CRE

\begin{tabular}{ll}
\hline Type of specimen & Number of specimens (\%) \\
\hline Urine & $90(59.2)$ \\
Exudates (pus, wound swab, sputum, BAL, deep tissue, ET & $76(50)$ \\
suction tip and aspirate, bile, bone tissue, and ear swab) & $22(14.1)$ \\
Blood & \\
\hline
\end{tabular}

BAL: Bronchoalveolar lavage, CRE: Carbapenem-resistant Enterobacteriaceae, ET: Endotracheal tube

Table 5: Different organisms isolated from the various clinical samples

\begin{tabular}{ll}
\hline Organism & Number (\%) \\
\hline Escherichia coli & $64(42.1)$ \\
Klebsiellapneumoniae & $60(39.4)$ \\
Citrobacter spp. & $14(9.2)$ \\
Enterobacter spp. & $14(9.2)$ \\
Proteus spp. & $3(1.9)$ \\
\hline
\end{tabular}

Table 6: Risk factors and comorbidities associated in patients with carbapenem-resistant isolates of Enterobacteriaceae

\begin{tabular}{lllll}
\hline Risk factors & $\begin{array}{l}\text { Number of patients with } \\
\text { CRE } \mathbf{n = 1 5 2}(\mathbf{\% )}\end{array}$ & $\begin{array}{l}\text { Number of patients with carbapenem } \\
\text { sensitive Enterobacteriaceae infection } \\
\mathbf{n = 3 1 0}(\mathbf{\%})\end{array}$ & Odds ratio & $\begin{array}{l}\text { p value (Chi-square } \\
\text { test) }\end{array}$ \\
\hline Catheterization & $142(93.4)$ & $196(63.2)$ & 8.08 & 0.000 \\
ICU stay & $126(82.8)$ & $181(58.3)$ & 12.323 & 0.000 \\
Mechanical ventilation & $142(93.4)$ & $209(67.4)$ & 6.714 & 0.000 \\
Prior surgery & $98(64.4)$ & $152(49)$ & 1.843 & 0.003 \\
Prior hospitalization & $110(72.3)$ & $168(54.3)$ & 2.165 & 0.000 \\
Prior antibiotic use & $114(75)$ & $146(47)$ & 3.297 & 0.000 \\
Malignancy & $32(21)$ & $19(5.1)$ & 2.703 & 0.000 \\
Diabetes mellitus & $40(26.3)$ & $73(23.5)$ & 1.112 & 0.67 \\
Hypertension & $57(37.5)$ & $96(30.9)$ & 1.15 & 0.54 \\
Acute/chronic renal disease & $65(42.7)$ & $83(27)$ & 1.69 & 0.01 \\
Liver disorder & $26(17.1)$ & $68(21.9)$ & 0.525 & 0.1 \\
Neurological disorder & $27(17.7)$ & $57(18.3)$ & 0.726 & 0.2 \\
Respiratory disease (COPD/BA) & $35(23)$ & $67(21.6)$ & 1.26 & 0.37 \\
Heart disease & $38(25)$ & $14(4.5)$ & 1.15 & 0.53 \\
HIV/AIDS & $10(6.5)$ & 1.26 & 0.66 \\
\hline
\end{tabular}

CRE: Carbapenem-resistant Enterobacteriaceae, ICU: Intensive care unit, COPD: Chronic obstructive pulmonary disease, BA: Bronchial asthma

CRE have emerged as a potential threat in hospitals with resistance to many other classes of antibiotics, thus limiting our therapeutic options. This situation demands proper diagnosis, treatment, and control of infections in hospitalized patients [12]. Global increase in CRE in many healthcare facilities poses challenges to infection control and infectious disease professionals. Risk-based screening is one useful strategy that has been used to limit the spread. We have demonstrated the value of understanding local epidemiology to help modify our riskbased screening as a strategy to limit the spread of CRE. The studies on transmission, mechanism of resistance, and treatment regimens are needed to control the spread of CRE.

\section{REFERENCES}

1. Xu Y, Gu B, Huang M, Liu H, Xu T, Xia W, et al. Epidemiology of carbapenem resistant Enterobacteriaceae (CRE) during 2000-2012 in
Asia. J Thorac Dis 2015;7(3):376-85.

2. Centers for Disease Control and Prevention (CDC). Vital signs: carbapenem-resistant Enterobacteriaceae. MMWR Morb Mortal Wkly Rep 2013;62(9):165-70.

3. Peirano G, Ahmed-Bentley J, Fuller J, Rubin JE, Pitout JD. Travelrelated carbapenemase-producing gram-negative bacteria in Alberta, Canada: the first 3 years. J Clin Microbiol 2014;52(5):1575-81.

4. Gupta N, Limbago BM, Patel JB, Kallen AJ. Carbapenem-resistant Enterobacteriaceae: epidemiology and prevention. Clin Infect Dis 2011;53(1):60-7.

5. Nordmann P, Naas T, Poirel L. Global spread of carbapenemaseproducing Enterobacteriaceae. Emerg Infect Dis 2011;17(10):1791-8.

6. Dasgupta S, Das S, Chawan NS, Hazra A. Nosocomial infections in the Intensive Care Unit: Incidence, risk factors, outcome and associated pathogens in a public tertiary teaching hospital of Eastern India. Indian J Crit Care Med 2015;19(1):14-20.

7. Swaminathan M, Sharma S, Poliansky Blash S, Patel G, Banach DB, 
Phillips M, et al. Prevalence and risk factors for acquisition of carbapenem-resistant Enterobacteriaceae in the setting of endemicity. Infect Control Hosp Epidemiol 2013;34(8):809-17.

8. Falagas ME, Rafailidis PI, Kofteridis D, Virtzili S, Chelvatzoglou FC, Papaioannou V, et al. Risk factors of carbapenem-resistant Klebsiella pneumoniae infections: A matched case control study. J Antimicrob Chemother 2007;60(5):1124-30.

9. Bhargava A, Hayakawa K, Silverman E, Haider S, Alluri KC, Datla S, et al. Risk factors for colonization due to carbapenemresistant Enterobacteriaceae among patients exposed to long-term acute care and acute care facilities. Infect Control Hosp Epidemiol 2014;35(4):398-405.

10. Ahmed-Bentley J, Chandran AU, Joffe AM, French D, Peirano G, Pitout JD. Gram-negative bacteria that produce carbapenemases causing death attributed to recent foreign hospitalization. Antimicrob Agents Chemother 2013;57(7):3085-91.

11. Lee GC, Lawson KA, Burgess DS. Clinical epidemiology of carbapenem-resistant Enterobacteriaceae in community hospitals: A case-case-control study. Ann Pharmacother 2013;47(9):1115-21.

12. Schwaber MJ, Klarfeld-Lidji S, Navon-Venezia S, Schwartz D, LeavittA, Carmeli Y. Predictors of carbapenem-resistant Klebsiella pneumoniae acquisition among hospitalized adults and effect of acquisition on mortality. Antimicrob Agents Chemother 2008;52(3):1028-33

13. ChitnisAS,CaruthersPS,RaoAK,LambJ,LurveyR,BeauDeRocharsV, et al. Outbreak of carbapenem-resistant Enterobacteriaceae at a long-term acute care hospital: Sustained reductions in transmission through active surveillance and targeted interventions. Infect Control Hosp Epidemiol 2012;33(10):984-92. 